It will be observed that the atomic weight of tellurium as given by use of 1909 figures for chlorine, bromine and potassium appears as 127.55 , which figure the author consiclers to be very close to the true ratio for tellurium.

UNIVERSITY OF WIBLONBIN, MADISON.

\title{
THE REACTION BETWEEN FUSED POTASSIUM NITRATE AND TELLURIUM DIOXIDE.
}

BI VICTOR I.ENHER AND P. I). POTTER.

Received October 17 , Igos.

In the preparation of telluric acid by the fusion of tellurium dioxide with potassium nitrate, there are two distinct stages: First, the transformation of the white dioxide into a yellow or brown substance which swims undissolved in the fused mass; second, the solution of this brown material in the fusion and its complete transformation to alkaline tellurate.

Berzelius 'has assumed this yellow substance to be a tetratellurate and describes it as insoluble in water, acids and alkalies. In the recent work of Baker and Bennet ${ }^{2}$ this same substance is described as a tetratellurate.

In preparing telluric acid by the fusion process in our laboratory this brown substance has been repeatedly observed. By examination with the microscope and by use of polarized light it appears homogeneous. It may be formed by heating potassium nitrate to $390^{\circ}-400^{\circ}$, the temperature at which oxygen is evolved from the fused salt, and adding finely powdered tellurium dioxide in small portions. The dioxide first turns yellow, then brown, and finally red-brown, all the while remaining undissolved. During these color changes considerable effervescence and loss of the oxides of nitrogen ensues. When the mass is allowed to cool, the substance appears yellow-brown. If, however, the process of heating be continued, the brown substance is further oxidized by the fused nitrate and the tellurium is completely converted into normal tellurate.

The brown substance appears, therefore, to be an intermediate product between tellurium dioxide and potassium tellurate.

Its composition is dependent upon the length of time of heating and on the quantities of nitrate and dioxide used. A series of intermediate products have been obtained by extracting such fusions with boiling water until no more soluble matter is removed, and analyzing the resulting substance.

Analysis.-In order to arrive at the composition of the brown material, the potassium content was determined by heating a weighed portion in a porcelain boat in a combustion tube in a current of hydrochloric acid gas and weighing the potassium chloride. The tellurium existing in the

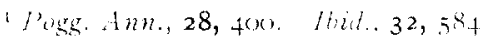

$\because$ I. (hem. Sue, $9 \mathrm{I}, \mathrm{I} 8+9$.
} 
form of trioxide was obtained by determining the active oxygen, as follows: The material was boiled with strong hydrochloric acid, the evolved chlorine conducted into potassium iodide and the liberated iodine titrated by means of a sodium thiosulphate solution. The total tellurium was determined by the method of Lenher and Homberger ${ }^{1}$ in either the portion which had been volatilized in the determination of the potassium or in the hydrochloric acid solution of the substance obtained in the determination of the active oxygen. Nitrogen was tested for very carefully and was found to be absent.

Experiment I.-Two grams of tellurium dioxide were heated with ten grams of potassium nitrate and the heating continued until a light yellow salt was formed. Analysis showed $\mathrm{TeO}_{3} \mathrm{I}_{3} .75, \mathrm{TeO}_{2} 82.93, \mathrm{~K}_{2} \mathrm{O} 3.04$, which would approximate the composition required by the formula: $2 \mathrm{~K}_{2} \mathrm{O}, 5 \mathrm{TeO}_{3}, 32 \mathrm{TeO}_{2}$.

Experiment II.-Conditions as in the previous experiment, except that less tellurium dioxide was used and the length of time of heating was longer. The material was yellow-brown in color, much darker than the first experiment. Analysis gave $\mathrm{TeO}_{3}$ I6.96, $\mathrm{TeO}_{2} \quad 78.57, \mathrm{~K}_{2} \mathrm{O}$ 4.23, which would indicate the existence of $\mathrm{K}_{2} \mathrm{O}_{2} \mathrm{TeO}_{3}$, I I TeO 2 .

Experiment $I I I$ was made with the view of obtaining a still more highly oxidized compound by longer fusion. Analysis indicated $\mathrm{TeO}_{3}$ 19.09, $\mathrm{TeO}_{2}$ 75.84, $\mathrm{K}_{2} \mathrm{O}$ 5.05, which corresponds to the formula $\mathrm{K}_{2} \mathrm{O}, 2 \mathrm{TeO}_{3}$, $9 \mathrm{TeO}_{2}$.

Properties. - These compounds are yellow brown in color, insoluble in water, in dilute acids and alkalis, but slowly dissolve in hot, strong hydrochloric acid with the evolution of chlorine and with the intermediate liberation of yellow tellurium trioxide. Heat alone breaks them down into alkaline tellurite and tellurium dioxide with evolution of oxygen. Fusion with potassium chlorate, sodium peroxide or with excess of potassium nitrate converts them to tellurate.

Sodium Salts.-By replacement of the potassium nitrate used by sodium nitrate, products of a similar character are obtained. Sodium nitrate when fused begins to evolve oxygen at about $350^{\circ}$ and when tellurium dioxide is added to the fused nitrate at this temperature, it quickly turns yellow, then red, the product formed being insoluble in the fused salts. On cooling, the substance appears as a red-brown powder. By varying the length of time of heating it has been possible to prepare two substances which on analysis showed the composition ${ }_{3} \mathrm{Na}_{2} \mathrm{O}, 4 \mathrm{TeO}_{3}$, ${ }_{25} \mathrm{TeO}_{2}$ and ${ }_{4} \mathrm{Na}_{2} \mathrm{O}, 5 \mathrm{TeO}_{3},{ }_{3} \mathrm{TeO}_{2}$. The general properties of these sodium compounds are similar to those of the potassium bodies.

Synthetical Experiments.-Believing from the analysis of many products obtained, that these brown substances are composed of alkaline tellurate

${ }^{1}$ This Journal, 30,387 . 
and tellurium dioxide, experiments were conducted with the view of ascertaining whether the brown salts could be obtained by direct union. To this end pure potassium tellurate was heated with tellurium dioxide in a platinum crucible. When the crucible was heated the mass was first converted into a yellow brown mass which darkened on further application of heat in a manner quite similar to the reaction between tellurium dioxide and potassium nitrate. On treating the cold mass with hot water a yellow-brown powder resulted which acted towards reagents exactly like the substances formed with the nitrates. When, however, potassium tellurate is heated alone in a platinum crucible, it fuses clear and colorless, and elevation of the temperature sufficient to cause part or all of the active oxygen to be expelled causes no formation of intermediate colored products.

Conclusions.-The composition of the products which are produced by heating tellurium dioxide with the alkaline nitrates depends largely on the length of time of heating. The first action is the oxidation of a portion of the tellurium dioxide to potassium tellurate, whereupon the tellurate formed unites with large amounts of tellurium dioxide with the formation of brown, insoluble bodies. These substances then lose tellurium dioxide by continuing the heating with the excess of nitrate until finally a clear fusion results and the tellurium has been completely converted into tellurate.

The formation of these intermediate products proceeds in a similar manner when sodium nitrate is substituted for the potassium salt, and the resulting products are of the same order of composition and properties.

Fusion with the chlorates yields the same character of compounds.

Synthesis of the potassium compounds from pure tellurate and tellurium dioxide seems to confirm the view that the compounds are intermediate products consisting of potassium tellurate and tellurium dioxide.

CheMICAL LABORATORY, UNIVERSity OF Wisconsin.

[CONTRIBLTION FROM THE JOHN HARRISON IABORATORY OF CHEMISTRY.]

\section{THE COLOR OF IODINE SOLUTIONS.}

By JOEL H. HILDEBRAND AND Ben I,EON GLAscock.

Iodine gives, as is well-known, different colored solutions in different solvents. With one group of solvents the color is violet, and with another it is yellow, red or brown, according to the solvent and the concentration. The cause of this difference has been the subject of considerable investigation. The earliest assumption ${ }^{1}$ was that the different colors were due to a difference in the molecular weight, the brown solutions containing more complex molecules, as $I_{4}$, while the violet solutions, like the vapor,

t Loeb, Z. physik. Chem, 2, 606 (1888). 